\title{
The Effects of Density, Spatial Pattern, and Competitive Symmetry on Size Variation in Simulated Plant Populations
}

\author{
J. Weiner, ${ }^{1, *}$ P. Stoll, ${ }^{2, \dagger}$ H. Muller-Landau, ${ }^{3, \neq}$ and A. Jasentuliyana ${ }^{4, \$}$
}

1. Department of Ecology, Royal Veterinary and Agricultural University, DK-1958 Frederiksberg, Denmark;

2. Geobotanical Institute, University of Bern, CH-3013 Bern, Switzerland;

3. Department of Ecology and Evolutionary Biology, Princeton University, Princeton, New Jersey 08544-1003;

4. Department of Ecology and Evolution, State University of New York, Stony Brook, New York 11794-5245

Submitted June 30, 2000; Accepted May 18, 2001

ABSTRACT: Patterns of size inequality in crowded plant populations are often taken to be indicative of the degree of size asymmetry of competition, but recent research suggests that some of the patterns attributed to size-asymmetric competition could be due to spatial structure. To investigate the theoretical relationships between plant density, spatial pattern, and competitive size asymmetry in determining size variation in crowded plant populations, we developed a spatially explicit, individual-based plant competition model based on overlapping zones of influence. The zone of influence of each plant is modeled as a circle, growing in two dimensions, and is allometrically related to plant biomass. The area of the circle represents resources potentially available to the plant, and plants compete for resources in areas in which they overlap. The size asymmetry of competition is reflected in the rules for dividing up the overlapping areas. Theoretical plant populations were grown in random and in perfectly uniform spatial patterns at four densities under size-asymmetric and size-symmetric competition. Both spatial pattern and size asymmetry contributed to size variation, but their relative importance varied greatly over density and over time. Early in stand development, spatial pattern was more important than the symmetry of competition in determining the degree of size variation within the population, but after plants grew and competition intensified, the size asymmetry of competition became a much more important

\footnotetext{
* E-mail: jw@kvl.dk.

† E-mail: stoll@sgi.unibe.ch.

${ }^{\ddagger}$ E-mail: helene@eno.princeton.edu.

`E-mail: ajasen@concentric.net.
}

Am. Nat. 2001. Vol. 158, pp. 438-450. (c) 2001 by The University of Chicago. 0003-0147/2001/15804-0009\$03.00. All rights reserved. source of size variation. Size variability was slightly higher at higher densities when competition was symmetric and plants were distributed nonuniformly in space. In a uniform spatial pattern, size variation increased with density only when competition was size asymmetric. Our results suggest that when competition is size asymmetric and intense, it will be more important in generating size variation than is local variation in density. Our results and the available data are consistent with the hypothesis that high levels of size inequality commonly observed within crowded plant populations are largely due to size-asymmetric competition, not to variation in local density.

Keywords: asymmetric competition, individual-based models, population structure, size inequality, spatial effects, zone of influence.

Competition among individuals usually increases size variation within plant populations, but there is controversy over the mechanisms through which this occurs. This controversy reflects a fundamental disagreement about the nature of competition among individual plants. Some studies have concluded that a major factor generating size variation in crowded plant populations is the "size asymmetry" of competition: larger plants have a disproportionate advantage (for their relative size) in competition with smaller plants, suppressing their growth (Begon 1984; Weiner 1990; Schwinning and Weiner 1998). In simple models, increases in size inequality with density are observed only when competition is size asymmetric (Weiner and Thomas 1986), so the commonly observed increase in size inequality in populations grown at higher densities has been considered evidence in support of the hypothesis of size-asymmetric competition (Weiner 1990).

Other researchers have argued that the importance of size-asymmetric competition has been exaggerated, and its existence has even been questioned (Turner and Rabinowitz 1983; Bonan 1991). Other phenomena, such as variation in exponential growth rates and variation in local crowding, might explain the patterns of size variation that have been attributed to size-asymmetric competition, such as the higher size inequality observed at higher densities. In spatially explicit models in which competition is local 
and individuals are growing in a random spatial pattern, variation in local crowding will generate variation in size, and this variation can increase at higher densities. For example, at very low densities there may be no competition, and plants will vary in size as a result of other factors. At somewhat higher densities, some plants will be competing (and therefore smaller) whereas others will not. This will result in greater size variation at this higher density, even if competition among competing individuals is completely size symmetric (Huston 1986; Miller and Weiner 1989).

Bonan (1991) took this argument farther, arguing that the higher size variability of plant populations grown at higher densities is not a reflection of competitive size asymmetry at all but rather of variation in local crowding, which exists because plants rarely grow in a uniform spatial pattern. In Bonan's spatially explicit model, size inequality increased with density under both symmetric and asymmetric competition when the spatial pattern was not uniform. He concluded that the commonly observed increase in plant size inequality at higher densities is not strong support for the existence of asymmetric competition.

To investigate the theoretical relationships between plant density, spatial pattern, and competitive asymmetry in determining size variation in crowded plant populations, we developed a spatially explicit, individual-based plant competition model based on overlapping zones of influence (Gates and Westcott 1978; Wyszomirski 1983). "Zone of influence" (ZOI) models, in which plants grow, occupy space, and compete for resources distributed in areas they overlap, are appealing because they are semimechanistic and conceptually simple, and they seem to capture some essential aspects of spatial competition among individual plants. ZOI models have other appealing attributes; since the definition of who is and who is not a neighbor falls directly out of the assumptions and behavior of the model, ZOI models avoid the problem of defining a "neighborhood size" (Stoll and Weiner 2000). Also, unlike many simulation approaches such as cellular automata (see, e.g., Ermentrout and Edelstein-Keshet 1993) or coupled lattice (Hendry et al. 1996) models, theoretical plants in ZOI models, like real plants, can grow over many scales, and their interactions scale accordingly. Finally, by making predictions concerning the influence of specific factors such as density, pattern, and mechanisms of competition on plant size distributions, ZOI models can be testable in a way that most general ecological models have not been (Weiner 1995).

Although conceptually simple, ZOI models are difficult to implement, except in the one-dimensional case, where space is represented by a line and competing plants are represented by overlapping line segments, or when twodimensional individuals are uniformly spaced and overlap only with their nearest neighbors (Gates and Westcott 1978; Gates et al. 1979; Gates 1982). Development of onedimensional ZOI models was pioneered by Wyszomirski (1986; Hara and Wyszomirski 1994). Wyszomirski (1986) showed that the size distributions produced by a ZOI model were similar to those of real plant populations, and his results emphasized the role of local spatial variation in generating plant size variability. Wyszomirski's original simulations did not include size-asymmetric competition, but a later study did (Hara and Wyszomirski 1994). It showed that spatial pattern was important in determining size variation when density was low and competition symmetric, but at higher density and asymmetric competition, spatial arrangement was much less important. Using a onedimensional ZOI model, Yastrebov (1996) demonstrated that symmetric competition leads to a type of stagnation, in which plants stop growing but do not die, whereas asymmetric competition results in growth of some plants and death (self-thinning) of others. He concluded that size asymmetry results in a more dynamic system.

Two-dimensional ZOI models have also been developed, originally for measuring interindividual competition in forest trees (Opie 1968; Bella 1971) and later as simulation models (Gates and Westcott 1978; Gates et al. 1979; Gates 1982; Wyszomirski 1983; Bonan 1988, 1991, 1993; Miller and Weiner 1989). Gates's ZOI model of tree plantations examined the effects of density and different degrees of competitive asymmetry in a uniform spatial pattern. Gates's model accounted for observed changes over time in the skewness of distribution of diameters in Pinus radiata plantations when competition was size asymmetric. Miller and Weiner (1989) investigated the effects of spatial pattern, density, and size asymmetry on size variation in a completely two-dimensional ZOI model. Size inequality increased with density if the spatial distribution of plants was random, but in the case of symmetric competition, this increase only occurred over a range of relatively low densities. Above a certain density, size inequality only increased with density if competition was asymmetric. In Bonan's (1991) model, size inequality was higher at higher densities for both modes of competition if the spatial pattern was not uniform. He concluded that "increased size variability [at higher densities] does not distinguish between symmetric and asymmetric resource distribution ... [but is] a direct manifestation of neighborhood competition" (Bonan 1991, p. 345).

Overall, two-dimensional ZOI studies to date have explored very small regions of the biologically relevant parameter space and have presented results for only a single point in time, which can give a distorted impression of the dynamic processes of stand development (Wyszomirski et al. 1999). Advances in computing technology and clarification of some of the central theoretical questions allow 
us to make more informative simulation studies. The question that emerges is, simply, what are the theoretical interactions between plant density, spatial arrangement, and size asymmetry of competition in determining variation in plant size during population development in a twodimensional ZOI model?

\section{Model}

In our ZOI model, plants grow as circles in two dimensions and can be thought of as ellipsoids in three dimensions. The area a plant occupies, $A$, represents resources potentially available to the plant and is allometrically related to the plant's biomass, $B$, as $A=c B^{2 / 3}$. A plant's potential growth, that is, its growth if there are no neighbors, is sigmoidal. Our criteria for selecting a growth equation were as follows: first, the positive term in the growth equation should be proportional to the resources available to the plant, which we assume scales with the area occupied; second, in the absence of competition, the equation should produce sigmoidal growth in biomass similar to that observed in plants; and third, the equation should be as simple as possible, with each parameter having a clear biological interpretation. Of the various functions that fit these criteria, we chose

$$
\frac{d B}{d t}=r\left(A-\frac{B^{2}}{B_{\max }^{4 / 3}}\right)=r\left(c B^{2 / 3}-\frac{B^{2}}{B_{\max }^{4 / 3}}\right),
$$

where $B_{\max }$ is the maximum (asymptotic) plant mass, $r$ is the initial (maximum) growth rate in mass per unit area occupied (in units of mass area ${ }^{-1}$ time $^{-1}$ ), and $t$ is time. The gain term is proportional to area occupied (as supported by West et al. [1999]), while the loss term is proportional to biomass squared as in the widely used logistic growth equation (Hunt 1982). Because we focus on the early stages of stand development, growth in our simulations is dominated by the gain term.

When plants overlap, they compete for resources in areas of overlap. The effective area of a plant $\left(A_{\mathrm{e}}\right)$ is the area it covers $(A)$ minus that part of the area lost to neighbors $\left(A_{\mathrm{n}}\right) . A_{\mathrm{e}}$ determines the realized growth rate of the plant during the next time interval:

$$
\frac{d B}{d t}=r\left(A_{\mathrm{e}}-\frac{B^{2}}{B_{\max }^{4 / 3}}\right)
$$

For simplicity in analyzing and interpreting the results, plants cannot have negative growth rates but continue to live and maintain the maximum size they achieve. Plants were grown in random and perfectly uniform spatial pat- terns. There is random normal independent variation in initial sizes $\left(B_{0}\right)$, initial relative growth rates $(r)$, and asymptotic sizes $\left(B_{\max }\right)$.

The size asymmetry of competition is reflected in the rules for dividing the overlapping areas among the competitors. The extreme case of "complete size asymmetry" (Schwinning and Weiner 1998) occurs when the larger individual obtains all the resources in the area of overlap. There are several possible types of more size-symmetric interactions (Gates et al. 1979; Wyszomirski 1986). Possibilities include dividing the area of overlap equally or in proportion to the relative sizes of the contestants. The question for the theoretical plant ecologist is, Which of these is the most biologically reasonable definition for size symmetry? Size asymmetry is usually defined in terms of plant size and overall resource division. If plants obtain resources in proportion to their size, this is considered to be size-symmetric competition, whereas if larger plants obtain a disproportional share of the resources (for their relative size), competition is considered size asymmetric (Schwinning and Weiner 1998). In a spatially explicit ZOI model, only resources in regions of overlap are contested; plants do not have access to, and therefore cannot compete for, resources beyond their zones. Thus, the usual definitions of size symmetry based on whole-plant resource acquisition or whole-plant competitive effects cannot be applied directly to a ZOI model, so we must look at the relevant biological principles. Schwinning and Fox (1995) argued that perfect size symmetry means equal uptake of resources per unit size. Similarly, Connolly and Wayne (1996) defined symmetry as equal competitive effects per unit size. According to this line of reasoning, symmetry in a ZOI model should mean that larger plants have no advantage per unit size within contested regions. Therefore, we define symmetry as the equal division of resources within areas of overlap. If two or more plants are overlapping the same area, resources in that area are divided equally among them, independent of their relative sizes.

This conservative definition of size-symmetric competition in a ZOI model is supported by the mathematics of overlapping circles of different sizes. When a large and a small circle overlap, the larger has a smaller percentage of its area in the region of overlap than does the smaller circle (Schwinning and Weiner 1998). Therefore, if the area of overlap is divided equally between them, the larger circle loses less of its area. Thus, there appears to be an inherent size asymmetry in a ZOI model, although this advantage may be counteracted when many plants are competing because larger plants will tend to overlap more neighbors than smaller plants. Dividing areas of overlap equally among all overlapping plants, and thus restricting the size advantage to the inherent advantage of being larger, is most consistent with the concept of symmetric competition as 
discussed in the recent literature. Equal division of contested resources is also the most symmetric of the alternatives, so that the results for less size-symmetric rules for dividing overlaps will lie between the results for this type of symmetry and those for totally asymmetric competition (Bonan 1988, 1991).

\section{Methods}

Because of the difficulty in analytically calculating areas overlapped by more than four circles, we used a discrete approximation of continuous two-dimensional space. In our implementation, space is divided into a fine grid. If the center of a grid cell is within a plant's area, the grid cell is considered to be covered by the plant; each grid cell can be covered by an unlimited number of plants. Runs presented below were performed with a grid of 25,000 cells. We performed tests of the sensitivity of the model to grid size and found the results to be robust. It is not clear how published studies have addressed this problem, and it is likely that the number of plants overlapping any point in space has been restricted in most or all previous studies.

Our model is similar to Bonan's $(1988,1991)$ but differs in the following respects: first, we use a discrete approximation of space (Bonan does not provide details on the implementation); second, we take a "wraparound" (torus) approach to avoiding edge effects rather than the "linear expansion factor" used by Bonan; and third, we use a different growth function. Also, Bonan created a "control" case in which competition was equal for all plants by setting the area lost to neighbors equal for all plants, whereas we consider the perfectly uniform pattern as the appropriate control for the comparison with the random spatial pattern. We also shift and expand greatly the parameter space investigated, although we still focus on the initial stages of stand development, during which plants are far from their asymptotic sizes. In Bonan's (1988) original study, density was not varied and was relatively low, such that competition was weak. In his second study (Bonan 1991), density was varied, but results were presented only for one point in time. Since competition increases in intensity as plants grow at a given density, we emphasize the changes that occur over time (Westoby 1982; Wyszomirski et al. 1999).

We assign conventional units to measures of mass and area for ease of interpretation; however, these units are essentially arbitrary and should not be taken literally. Initial masses $\left(B_{0}\right)$ were distributed normally with a mean of $1.0 \mathrm{mg}$ and a standard deviation of 0.1. Maximum size $\left(B_{\max }\right)$ was $20,000 \mathrm{mg}$ with a standard deviation of 2,000 . The area, $A$, of each plant (in $\mathrm{cm}^{2}$ ) was set equal to its mass, $B$ (in $\mathrm{mg}$ ), raised to the $2 / 3$ power; thus, initial plant areas averaged $1.0 \mathrm{~cm}^{2}$ and maximal plant areas averaged $159 \mathrm{~cm}^{2}$. The virtual plot on which simulations took place had an area of 10,000 $\mathrm{cm}^{2}$ (with grid cells of size $0.2 \mathrm{~cm}^{2}$ ). Initial (i.e., maximum) growth rates $(r)$ were distributed with a mean of $1.0 \mathrm{mg} \mathrm{cm}{ }^{-2}$ time step ${ }^{-1}$ (where the area is the effective area from which the plant is able to draw resources) and a standard deviation of 0.1 . Theoretical populations were grown for 30 time steps at four densities, $100,506,992$, or 4,970 plants $\mathrm{m}^{-2}$; in two spatial patterns, random or uniform (approximately hexagonal); and with two modes of competition, size symmetric or size asymmetric.

The uniform spatial pattern deviated from perfectly hexagonal to accommodate a square plot; neighbors in one direction were up to $13 \%$ closer than neighbors in the other two directions. The pattern is perfectly uniform since all plants experienced exactly the same configuration of neighbors and is therefore an appropriate control for spatial variation. For simplicity, we present extensive data for only two of the densities, 100 ("low") and 992 ("high").

We also investigated one case of "partial size asymmetry," in which areas of overlap are divided in proportion to the relative biomasses of the overlapping individuals. As expected, the results were intermediate between size symmetric and asymmetric, and, in the interests of brevity, we do not present them here.

The output of the model consists of the sizes and locations of all the individual plants over time (see fig. 1). We analyzed the distribution of individual masses, the behavior of mean mass and size inequality (coefficient of variation $[\mathrm{CV}]$ of mass), and the relationships between the size of individuals at the beginning of a size interval and their growth over the interval. Such relationships, which have been called "distribution modifying functions" (Westoby 1982), " $G[t, x]$ " functions (Hara 1984), or simply "growth-size relationships" (Weiner 1990), describe how a size distribution changes over time.

To test the effects of initial conditions and stochastic variation on the results, selected model runs were repeated 10 times with different random number seeds. Variation among such "replicates" was so small that replication of the remaining runs was deemed unnecessary.

The complete code of our model (in C) is available on the Web at http://www.ecol.kvl.dk/admin/php3/researchbot.php3. The program is menu-driven and thus userfriendly, and we invite researchers to use it for further studies.

\section{Results \\ Mean Size}

After 20 time steps, there was still no competition at the lowest density in the uniform pattern, whereas competi- 


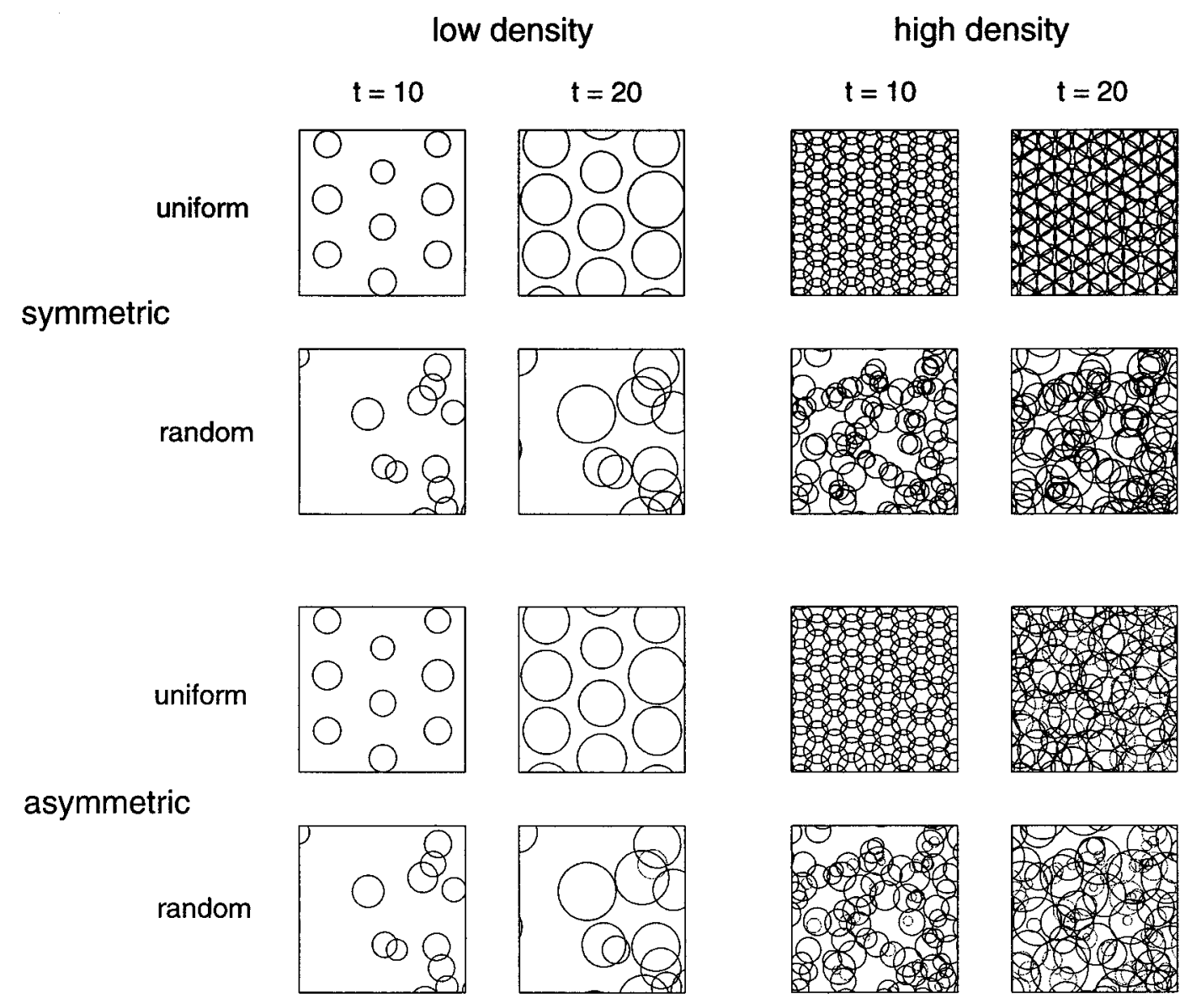

Figure 1: Subplots at $t=10$ and 20, at low (100) and high (992) density, growing under asymmetric and symmetric competition, in random and uniform patterns. Plants with growth rate of 0 are shown with a dashed outline.

tion was quite intense at high densities (fig. 1). Mean mass was lower at higher densities and slightly lower in a random than in a uniform pattern (fig. 2). Mean mass was also slightly lower under symmetric than asymmetric competition, and this effect increased with time and at higher densities.

\section{Size Inequality}

Size inequality (CV of mass) was higher when competition was asymmetric than when it was symmetric and higher in a random than in a uniform spatial pattern (fig. $3 A$, $3 B)$. Size inequality increased strongly with density when competition was asymmetric and increased when competition was symmetric and the spatial pattern was random. When competition was symmetric and the spatial arrangement uniform, size inequality decreased slightly with density (fig. $3 A, 3 B$ ).
At low densities and early in growth, spatial pattern played a more important role than size asymmetry in generating size variability (fig. $3 A$ ), but at high densities or after longer periods of growth, the size asymmetry of competition was more important than spatial pattern (figs. 3, $4 A$ ). At high densities or after a longer period of growth, the contribution of spatial pattern to size inequality, that is, the difference in CV between a uniform and a random pattern, was greater under symmetric than under asymmetric competition if the comparison is made at the same point in time (fig. 4A). But the absolute contribution of spatial pattern to size variation was very similar for symmetric and asymmetric competition when populations are compared at the same mean plant biomass (fig. $4 B$ ). The relative contribution of spatial pattern to size variation decreases with time and density as the asymmetry of competition comes to dominate the generation of size variation (figs. 3, 4A). 


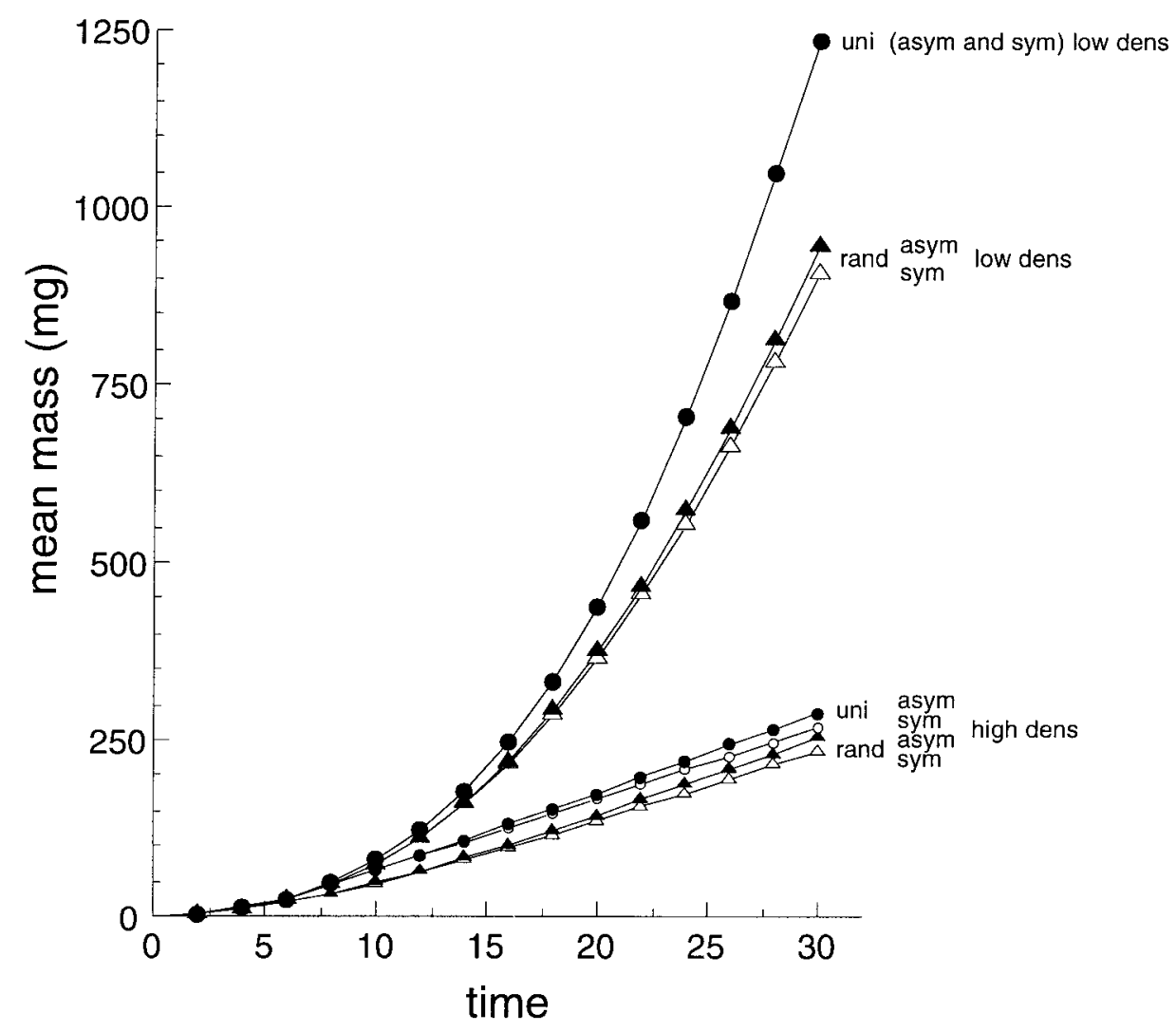

Figure 2: Mean plant mass over time for theoretical plant populations growing in uniform (circles) and random (triangles) patterns, under sizesymmetric (open symbols) and size-asymmetric (filled symbols) competition, at low (100 plants; large symbols) and high (992 plants; small symbols) density.

\section{Size Distributions and Growth-Size Relationships}

When competition is asymmetric, small plants that are covered by larger neighbors do not grow at all, and a lower tail or mode of individuals that are not growing develops (fig. 5L-5P). After asymmetric competition became intense, size variation increased steeply (fig. $4 A$ ). Under symmetric competition, all plants grew more slowly, but all plants grew somewhat if any grew.

The relationships between size at a given point in time and subsequent growth were very different for symmetric and asymmetric competition, and this difference increased with time (fig. 6). When plants are in the early stages of growth and there is no competition, growth is an approximately linear function of size (fig. $6 A, 6 I$ ) because variation in both size and growth are determined primarily by variation in $r$ and because both have the same dependence on $r$. In a uniform pattern at high density, the slope of this relationship is reduced by competition, but it remains approximately linear (fig. 6C, 6D). In a random pattern with symmetric competition, the growth-size re- lationship curves upward slightly, and variation in growth rate increases with increasing size (fig. 6G,6H). Asymmetric competition generates a large number of nongrowing plants and a group of large plants that are not affected by competition (fig. $6 K-6 P$ ). Spatial pattern affected growth-size relationships when competition was symmetric, but its influence under asymmetric competition decreased over time; later in stand development, growth-size relationships under asymmetric competition look similar for both spatial patterns (fig. $6 L, 6 P$ ).

\section{Discussion}

Density, spatial pattern, and the degree of size asymmetry all affect the amount of size variation in our theoretical plant populations. These factors interact such that their relative contributions to population size variability vary greatly within parameter space and over time as individuals grow and compete. 


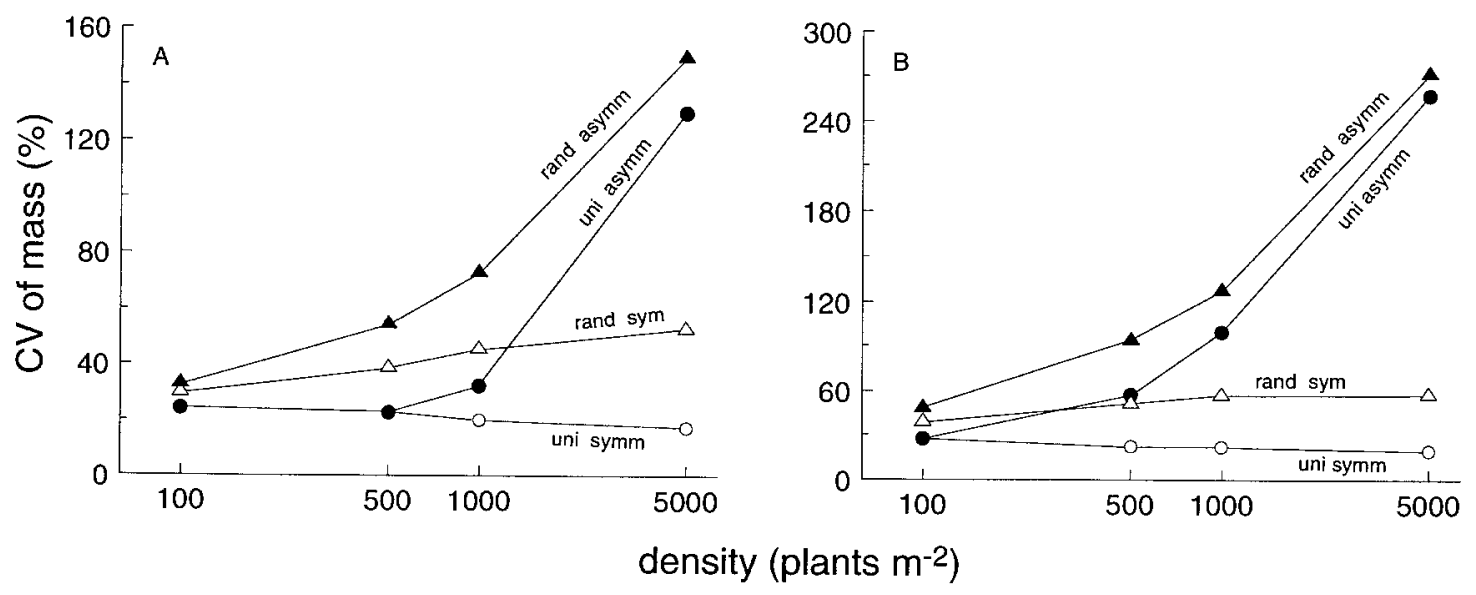

Figure 3: Coefficient of variation of mass versus density at time $=10(A)$ and $20(B)$ for theoretical plant populations growing in uniform (circles) and random (triangles) spatial patterns, with size-symmetric (open symbols) and size-asymmetric (filled symbols) division of overlapping zones of influence.

General Effects of Spatial Pattern and the Degree of Size Asymmetry on Size Variation

As intuition suggests, and as previous studies have demonstrated, competitive asymmetry will always increase size variation over what it would be under more symmetric competition because if competition is asymmetric, large plants suppress the growth of their smaller neighbors, increasing size differences. The effect of asymmetry on size variation increases with the intensity of competition (i.e., at higher density or larger mean plant size). When asymmetric competition becomes very intense, only larger plants can continue to grow, while their smaller neighbors are completely suppressed (fig. 6). It is not always appreciated that in the theoretical case of completely sizeasymmetric competition, the very largest plants do not suffer competition at all; they grow exactly as they would with no neighbors at all. This may be rare in nature, although there is some evidence that it can occur (Thomas and Weiner $1989 a, 1989 b$ ).

Size variation is always higher when plants are growing in a random as opposed to a uniform pattern, but the strength of this effect varies within the parameter space we explored (discussed below). Many of the effects of spatial pattern in the model are due to differences in the onset and development of competition. In a uniform spatial pattern, all plants grow without any competition for an initial period of time, and then competition commences for all plants over a short period (fig. 1, third column). In a random pattern, competition begins immediately for some individuals, whereas other individuals may grow for a relatively long period without experiencing competition (fig. 1, first column). Thus, a uniform pattern results in in- creased biomass (fig. 2) since plants grow without competition for as long as possible, and the ground is covered as quickly as possible (fig. 1, third column).

In a ZOI model in which plants are still in the early phase of growth, the effect of density is similar to the effect of time, the difference being primarily one of scale. This can be observed by the similar effects of density (fig. 3A) and time (fig. $4 A$ ) on size variation. Plant density is only meaningful in relation to mean plant size. Raising the density increases the overall intensity of competition, defined as the reduction in mean performance due to neighbors, as does increased plant size at a given density. The behavior of the model at high density is very similar to its behavior at low density at a later period of time, when mean plant size is much larger. Many phenomena are better analyzed in terms of mean plant size than in terms of time, although most experimental data are collected after a given period of time.

\section{Interactions between Density, Spatial Pattern, and Competitive Symmetry}

Our results support the hypothesis (Wyszomirski 1986) that spatial pattern can contribute to the increase in size variation observed at higher densities and that symmetric competition among plants distributed nonuniformly in space can produce patterns that have been attributed to asymmetric competition (Miller and Weiner 1989; Bonan 1991). For example, a growth-size relationship with an increasing slope, usually considered evidence for asymmetric competition (Weiner 1990), can occur under symmetric competition if spatial pattern is not uniform (fig. 


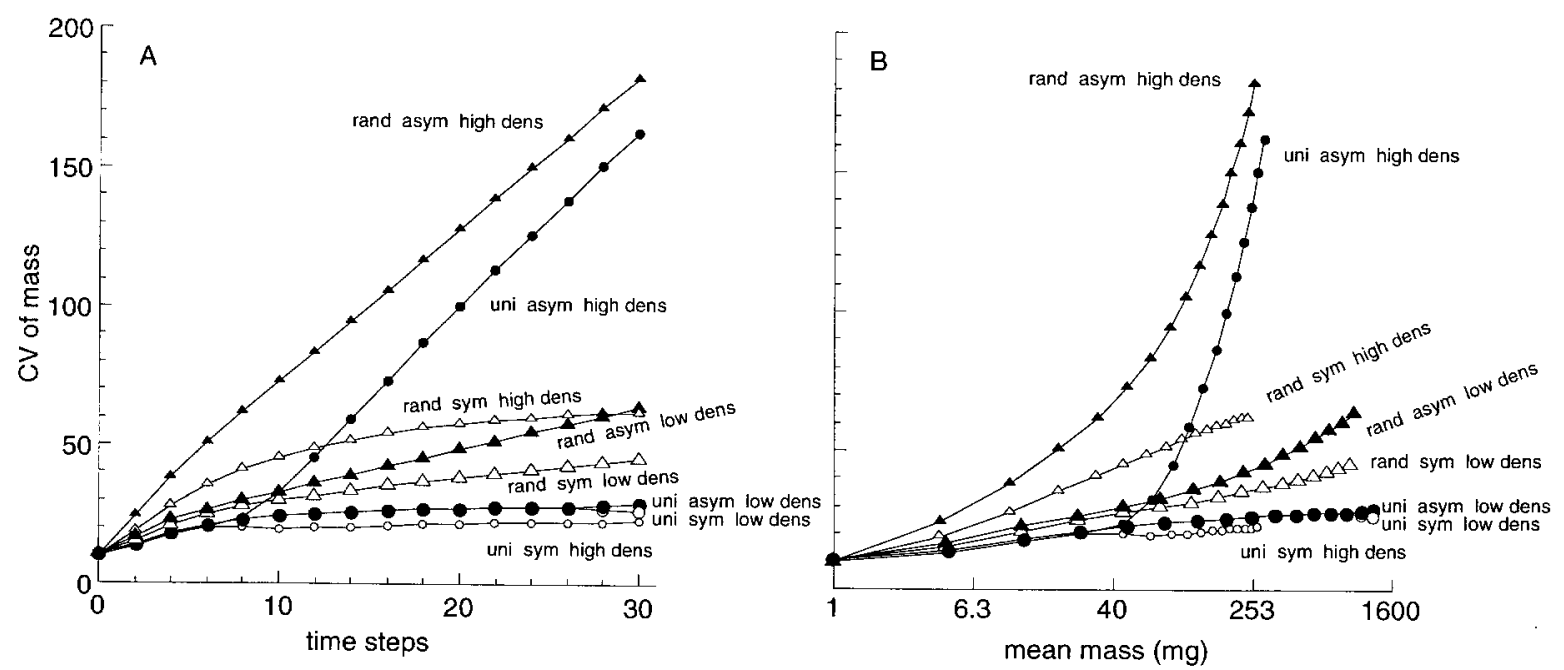

Figure 4: Size inequality (coefficient of variation) versus $(A)$ time and $(B) \log$ mean mass for theoretical plant populations growing in uniform (circles) and random (triangles) patterns, under size-symmetric (open symbols) and size-asymmetric (filled symbols) competition, at a low (100 plants; large symbols) and high (992 plants; small symbols) density. The sequence of points reflects intervals of two steps in the model, from $t=0$ to $t=30$.

$6 G, 6 H)$. Similarly, increased size variation in populations grown at higher density, usually considered evidence for competitive asymmetry, can occur under symmetric competition when the spatial pattern is nonuniform (fig. $3 A$ ). This effect appears to be weak, however, and becomes even weaker at higher densities and later in growth (fig. 3B).

By reducing the growth rates of competing plants in a somewhat equal way, symmetric competition will tend to reduce the divergence in sizes generated by variation in initial growth rates (Koyama and Kira 1956; Wyszomirski et al. 1999). Thus, in nonspatial models (see, e.g., Weiner and Thomas 1986) or in a uniform pattern (fig. 3), increasing the density results in smaller, more equally sized plants. When the spatial pattern is not uniform, some plants will begin competing with their neighbors before others. Even if this competition is symmetric and makes those early-competing plants smaller and more equal to each other, it will increase the difference in size between them and those plants that are not yet experiencing competition (Miller and Weiner 1989). It is this variation in the onset and development of competition that increases size variation under symmetric competition. At higher densities, this variation in local competitive environments starts earlier, and resulting differences are propagated longer, which results in greater size variation at higher densities under symmetric, as well as asymmetric, competition. The inherent asymmetry of a ZOI model at the individual level, discussed in the "Model" section, could also play a role.

When symmetrically competing plants are uniformly distributed in space, size variation is lower at higher densities (fig. 3). Thus, our results suggest that observed increases in size variability at higher densities provide strong support for the hypothesis that competition is asymmetric if the spatial pattern is uniform. Many of the experiments showing increased size variation at higher densities have, in fact, been performed on populations that were hyperdispersed for experimental purposes (see, e.g., Edmeades and Daynard 1979; Weiner 1985).

Spatial effects are relatively important when competition is symmetric and/or weak (see, e.g., Stoll et al. 1994), but spatial effects become less important when competition is strong and highly asymmetric (Hara and Wyszomirski 1994). Spatial arrangement is less important when competition is strongly asymmetric because larger plants will dominate their smaller neighbors when they begin to compete, irrespective of the exact distance to or number of the smaller neighbors. After competition begins in an asymmetrically competing population, the size variation generated by asymmetry develops very quickly, and this size variation approaches that of a randomly distributed asymmetrically competing population (fig. $4 A$ ).

The effects of the spatial pattern on size variation are reduced under asymmetric competition because the distribution of growing plants in the uniform pattern becomes nonuniform as some plants are completely suppressed (fig. 1), whereas the spatial distribution of growing plants in a random spatial pattern becomes more uniform with time (P. Stoll, unpublished results), as observed in plant populations during the process of self-thinning 


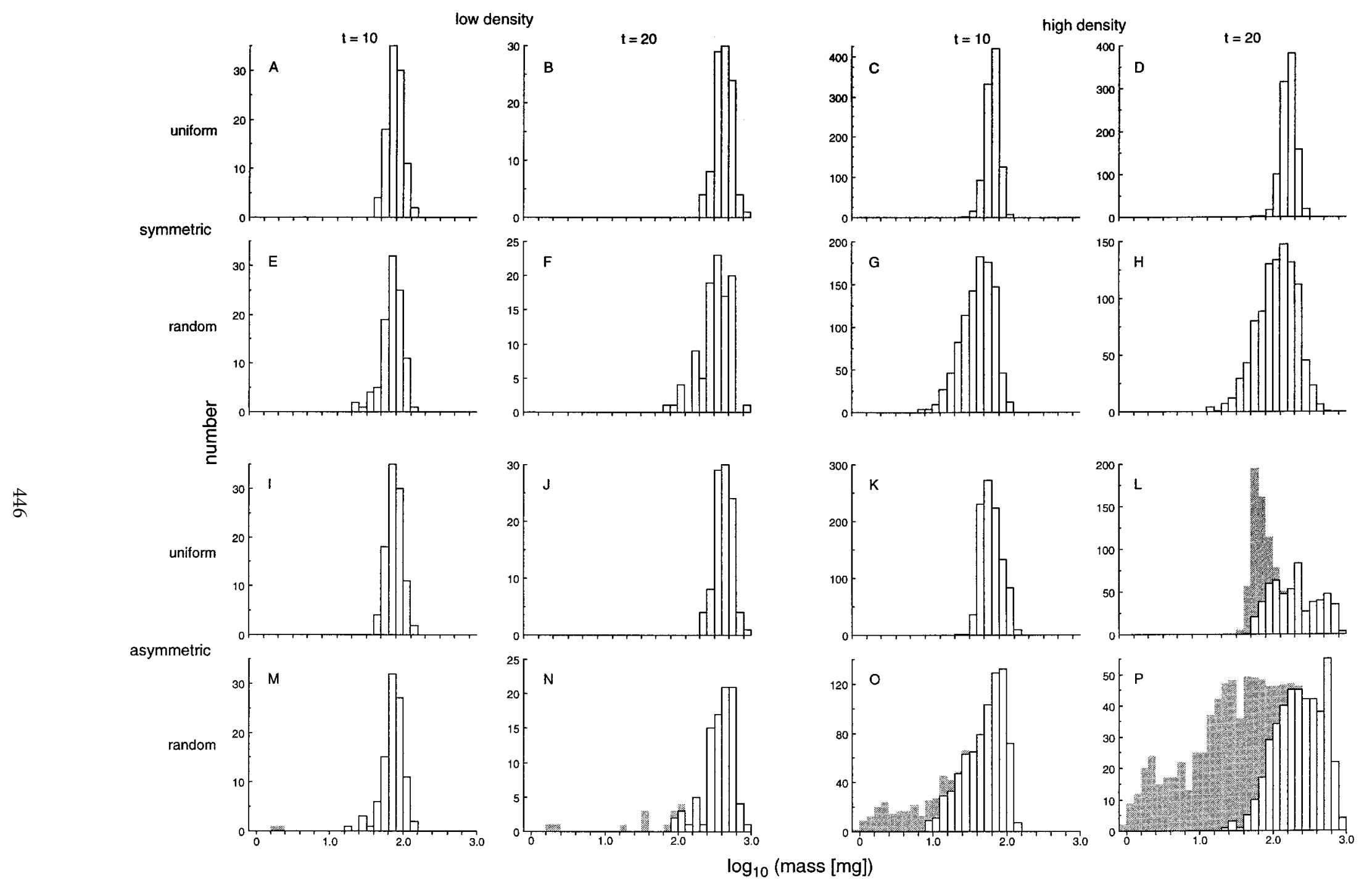

Figure 5: Size distributions for populations growing at low (100; left half) and high (992; right half) density, in uniform and random spatial patterns, under symmetric (A-H) and asymmetric $(I-P)$ competition at $t=10$ and $t=20$ (left and right column within each density). Plants with growth rate of 0 are shown as gray bars. 


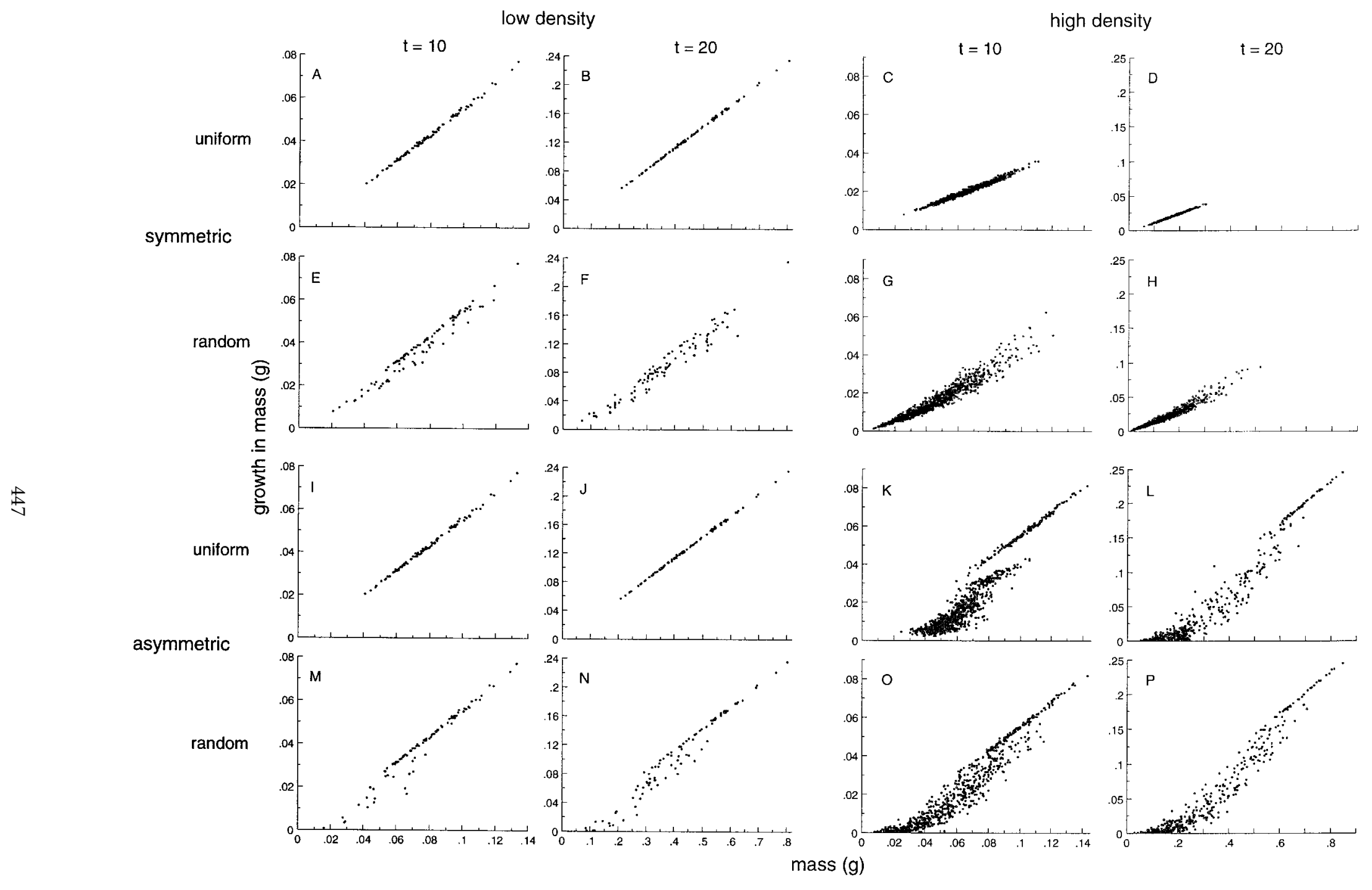

Figure 6: Change in mass (absolute growth rate) over the period $t=10$ to $t=12$ and $t=20$ to $t=22$, versus size at $t=10$ and 20, respectively, for plants grown at low (100) and high (992) density in uniform and random spatial patterns, under symmetric $(A-H)$ and asymmetric $(I-P)$ competition. 
(Kenkel 1988). Under symmetric competition, all individuals can grow somewhat if any can grow, so spatial pattern always plays a role.

\section{Reassessing the Role of Local Spatial Pattern in Generating Plant Size Variation}

Neither our results nor the available empirical data support the great emphasis that some theoreticians have placed on the role of small-scale spatial pattern in generating size inequality among competing plants (e.g., Huston and DeAngelis 1987; Bonan 1991). Although plants grown experimentally in nonuniform spatial patterns often do show greater size inequality than plants grown in uniform patterns, these differences are usually much smaller than would be expected from models that focus primarily on two-dimensional spatial pattern. There was significantly higher size inequality in randomly sown than in uniformly sown populations of Trifolium incarnatum and Lolium multiflorum in only one of four cases, and in one of the cases inequality was actually higher in the hexagonal pattern (Weiner 1985). There was a significant effect of spatial pattern (hexagonal vs. random vs. clumped) in Prunella vulgaris populations grown at 308 and 769 plants $\mathrm{m}^{-2}$ but not at 3,077 plants $\mathrm{m}^{-2}$ (Miller et al. 1994). Such experimental results argue strongly for the importance of competitive asymmetry in generating size variation at high densities. In our model, if competition is totally asymmetric, its role in generating size variation will increase with density and over time, while the relative contribution of spatial pattern decreases accordingly. Local variation in crowding seems to be most important in generating size variation when competition is symmetric (see, e.g., Weiner 1984; Stoll et al. 1994), but investigations of intermediate levels of size asymmetry are needed.

The effects of spatial pattern on size variation are also smaller in the glasshouse or field than in most models because plasticity in growth form means that a plant's location is not totally fixed by its point of emergence from the soil (Weiner 1985). For example, the locations of growing apices on plants that have a main axis may be much more uniform than the locations of their bases because plants grow toward the light and thus try to avoid neighbors (Sorrensen-Cothern et al. 1993; Umeki 1997). Plasticity in growth alters the spatial pattern and reduces the intensity and asymmetry of competition (Schwinning and Weiner 1998). In our model, a zero growth rate due to competition occurs immediately for some plants in asymmetrically competing, nonuniformly distributed populations. When locations are random or clumped in space, some plants will have very close neighbors. If competition is asymmetric, a small plant will stop growing or die as soon as it is completely covered by one or more larger individuals. This does not happen in the field because plasticity allows plants to adjust their locations somewhat and because competition is rarely completely size asymmetric. What most limits the usefulness of ZOI models may not be their simple mechanism of competition but the absence of plasticity. Plasticity could be incorporated in future studies.

\section{The Problem of Death in Analyzing Size Distributions}

Death complicates the analysis of plant size distributions. The results of most studies of plant population development are contingent on survival. Density-dependent mortality (self-thinning) occurs when crowding is severe, and it is concentrated among the smallest individuals, thus altering the size distribution. As a crowded stand develops, size inequality increases until the onset of self-thinning. During self-thinning, size inequality of survivors decreases, and after extensive mortality, the relatively few survivors are quite equal in size (Weiner and Thomas 1986; Weiner 1988; Kenkel et al. 1997). If, however, one were to consider the size distribution of all individuals, living and dead (by considering the size of dead plants to be their size at death), size inequality would increase monotonically during selfthinning (Thomas and Weiner 1989a). Thus, there may be cases in which plants grown at higher density show lower size inequality than plants grown at lower density if one looks only at survivors.

Whether or not to include dead individuals in the analysis of plant size distributions depends on the question being asked. If the question concerns biomass accumulation, dead plants might be best considered to have the size that they achieved before dying, as we have done here, but if the question of interest is fecundity distributions in monocarpic plants, dead plants may be considered to have a value of 0 if they die before reproducing.

\section{Conclusion}

In this study, we have used a ZOI model to clarify the relative importance of density, spatial pattern, and competitive symmetry to size variation in simple plant populations. Although we have expanded the parameter space investigated greatly over previous studies, we have remained in a relatively restricted region so that our results could be compared to other models and available data. Further questions that arise immediately include the effects of different growth equations, intermediate degrees of size asymmetry, clumped spatial patterns, and increased stochastic variation in parameter values. The model can also be used to look at much later stages of population development, such as self-thinning. Future work should combine modeling with empirical studies that inform 
choices of equations and parameters in the model and also allow for direct comparison of model and field results (see, e.g., Berger and Hildenbrandt 2000). ZOI models have much to offer plant population biology, both as theoretical tools and in empirical studies.

\section{Acknowledgments}

This research was supported by Swarthmore College, a Bullard Fellowship from Harvard Forest (to J.W.), and postdoctoral fellowships from the Swiss National Science Foundation and Roche Research Foundation (to P.S.). We thank J. Bastow Wilson, T. E. Miller, S. Schwinning, S. D. Wilson, T. Wyszomirski, and an anonymous reviewer for helpful comments on the manuscript and F. Amador for starting to build this model many years ago.

\section{Literature Cited}

Begon, M. 1984. Density and individual fitness: asymmetric competition. Pages 175-194 in B. Shorrocks, ed. Evolutionary ecology. Blackwell Scientific, Oxford.

Bella, I. E. 1971. A new competition model for individual trees. Forest Science 17:364-372.

Berger, U., and H. Hildenbrandt. 2000. A new approach to spatially explicit modelling of forest dynamics: spacing, ageing and neighbourhood competition of mangrove trees. Ecological Modelling 132:287-302.

Bonan, G. B. 1988. The size structure of theoretical plant populations: spatial patterns and neighborhood effects. Ecology 69:1721-1730.

- 1991. Density effects on the size structure of annual plant populations: an indication of neighbourhood competition. Annals of Botany (London) 68:341-347.

- 1993. Analysis of neighborhood competition among annual plants: implications of a plant growth model. Ecological Modelling 65:123-136.

Connolly, J., and P. Wayne. 1996. Asymmetric competition between plant species. Oecologia (Berlin) 108:311-320.

Edmeades, G. O., and T. B. Daynard. 1979. The development of plant-to-plant variability in maize at different planting densities. Canadian Journal of Plant Science 59:561-576.

Ermentrout, G. B., and L. Edelstein-Keshet. 1993. Cellular automata approaches to biological modelling. Iournal of Theoretical Biology 160:97-133.

Gates, D. J. 1982. Competition and skewness in plantations. Journal of Theoretical Biology 94:909-922.

Gates, D. J., and M. Westcott. 1978. Zone of influence models for competition in plantations. Advances in Applied Probability 10:299-537.

Gates, D. J., A. J. O'Connor, and M. Westcott. 1979. Partitioning the union of disks in plant competition mod- els. Proceedings of the Roval Societv of London A 367: 59-79.

Hara, T. 1984. A stochastic model and the moment dynamics of the growth and size distribution in plant populations. Journal of Theoretical Biology 109:173-190.

Hara, T., and T. Wyszomirski. 1994. Competitive asymmetry reduces spatial effects on size-structure dynamics in plant populations. Annals of Botanv (London) 73: 285-297.

Hendry, R. J., J. M. McGlade, and J. Weiner. 1996. A coupled map lattice model of the growth of plant monocultures. Ecological Modelling 84:81-90.

Hunt, R. 1982. Plant growth curves: the functional approach to plant growth analysis. Edward Arnold, London.

Huston, M. 1986. Size bimodality in plant populations: an alternative hypothesis. Ecology 67:265-269.

Huston, M., and C. S. G. DeAngelis. 1987. Size bimodality in monospecific populations: a critical review of potential mechanisms. American Naturalist 129:678-707.

Kenkel, N. C. 1988. Pattern of self-thinning in jack pine: testing the random mortality hypothesis. Ecology 69: 1017-1024.

Kenkel, N. C., M. L. Hendrie, and I. E. Bella. 1997. A longterm study of Pinus banksiana population dynamics. Journal of Vegetation Science 8:241-254.

Koyama, H., and T. Kira. 1956. Intraspecific competition among higher plants. VIII. Frequency distribution of individual plant weight as affected by the interaction between plants. Journal of the Institute of Polytechnics, Osaka City University 7:73-94.

Miller, T. E., and J. Weiner. 1989. Local density variation may mimic effects of asymmetric competition on plant size variability. Ecology 70:1188-1191.

Miller, T. E., A. A. Winn, and D. W. Schemske. 1994. The effects of density and spatial distribution on selection for emergence time in Prunella vulgaris. American Journal of Botany 81:1-6.

Opie, J. E. 1968. Predictability of individual tree growth using various definitions of competing basal area. Forest Science 14:314-323.

Schwinning, S., and G. A. Fox. 1995. Population dynamic consequences of competitive symmetry in annual plants. Oikos 72:422-432.

Schwinning, S., and J. Weiner. 1998. Mechanisms determining the degree of size-asymmetry in competition among plants. Oecologia (Berlin) 113:447-455.

Sorrensen-Cothern, K. A., E. D. Ford, and D. G. Sprugel. 1993. A model of competition incorporating plasticity through modular foliage and crown development. Ecological Monographs 63:277-304.

Stoll, P., and J. Weiner. 2000. A neighbourhood view of interactions among individual plants. Pages 11-27 in U. 
Dieckmann, R. Law, and J. A. J. Metz, eds. The geometry of ecological interactions: simplifying spatial complexity. Cambridge University Press, Cambridge.

Stoll, P., J. Weiner, and B. Schmid. 1994. Growth variation in a naturally established population of Pinus sylvestris. Ecology 75:660-670.

Thomas, S. C., and J. Weiner. 1989a. Growth, death and size distribution change in an Impatiens pallida population. Journal of Ecology 77:524-536.

. $1989 b$. Including competitive asymmetry in measures of local interference in plant populations. Oecologia (Berlin) 80:349-355.

Turner, M. D., and D. Rabinowitz. 1983. Factors affecting frequency distributions of plant mass: the absence of dominance and suppression in Festuca paradoxa. Ecology 64:469-475.

Umeki, K. 1997. Effect of crown asymmetry on size-structure dynamics of plant populations. Annals of Botany (London) 79:631-641.

Weiner, J. 1984. Neighborhood interference amongst Pinus rigida individuals. Journal of Ecology 72:183-195.

-1985. Size hierarchies in experimental populations of annual plants. Ecology 66:743-752.

. 1988. Variation in the performance of individuals in plant populations. Pages 59-81 in A. J. Davy, M. J. Hutchings, and A. R. Watkinson, eds. Plant population ecology. Blackwell Scientific, Oxford.
1990. Asymmetric competition in plant populations. Trends in Ecology \& Evolution 5:360-364.

-1995. On the practice of ecology. Journal of Ecology 83:153-158.

Weiner, J., and S. C. Thomas. 1986. Size variability and competition in plant monocultures. Oikos 47:211-222.

West, G. B., J. H. Brown, and B. J. Enquist. 1999. A general model for the structure and allometry of plant vascular systems. Nature (London) 400:664-667.

Westoby, M. 1982. Frequency distributions of plant size during competitive growth of stands: the operation of distribution-modifying functions. Annals of Botany (London) 50:733-735.

Wyszomirski, T. 1983. A simulation model of the growth of competing individuals of a plant population. Ekologia Polska 31:73-92.

- 1986. Growth, competition and skewness in a population of one-dimensional individuals. Ekologia Polska 34:615-641.

Wyszomirski, T., I. Wyszomirski, and I. Jarzyna. 1999. Simple mechanisms of size distribution dynamics in crowded and uncrowded virtual monocultures. Ecological Modelling 115:253-273.

Yastrebov, A. B. 1996. Different types of heterogeneity and plant competition in monospecific stands. Oikos 75: 89-97.

Associate Editor: Scott D. Wilson 